\title{
Tissue Inhibitor of Matrix Metalloproteinases-1 Knockdown Suppresses the Proliferation of Human Adipose-Derived Stem Cells
}

\author{
Peihua Zhang, ${ }^{1}$ Jin Li, ${ }^{1}$ Yawei Qi, ${ }^{1}$ Xudong Tang, ${ }^{2}$ Jianfeng Duan, ${ }^{1}$ Li Liu,, ${ }^{1}$ Zeyong Wu, \\ Jie Liang, ${ }^{1}$ Jiangfeng Li, ${ }^{1}$ Xian Wang, ${ }^{1}$ Guofang Zeng, ${ }^{1}$ and Hongwei Liu ${ }^{3}$ \\ ${ }^{1}$ Institute of Plastic Surgery, Stem Cell Research and Clinical Translation Center, Affiliated Hospital of Guangdong Medical University, \\ Zhanjiang, Guangdong 524001, China \\ ${ }^{2}$ Institute of Biochemistry and Molecular Biology, Guangdong Medical University, Zhanjiang, Guangdong 524023, China \\ ${ }^{3}$ Department of Plastic Surgery, The First Affiliated Hospital of Jinan University, Key Laboratory for Regenerative Medicine, \\ Ministry of Education, Guangzhou, Guangdong 510630, China
}

Correspondence should be addressed to Guofang Zeng; guofangz@yahoo.com and Hongwei Liu; liuhongwei0521@hotmail.com

Received 28 December 2015; Accepted 10 April 2016

Academic Editor: Franca Fagioli

Copyright (C) 2016 Peihua Zhang et al. This is an open access article distributed under the Creative Commons Attribution License, which permits unrestricted use, distribution, and reproduction in any medium, provided the original work is properly cited.

\begin{abstract}
Tissue inhibitor of metalloproteinases-1 (TIMP-1) is a multifunctional matrix metalloproteinase, and it is involved in the regulation of cell proliferation and apoptosis in various cell types. However, little is known about the effect of TIMP-1 expression on the proliferation of adipose-derived stem cells (ADSCs). Therefore, TIMP-1 expression in the ADSCs was firstly detected by western blotting, and TIMP-1 gene was knocked down by lentivirus-mediated shRNA. Cell proliferation was then evaluated by MTT assay and Ki67 staining, respectively. Cell cycle progression was determined by flow cytometry. The changes of p51, p21, cyclin E, cyclindependent kinase 2 (CDK2), and P-CDK2 caused by TIMP-1 knockdown were detected by western blotting. The results indicated that ADSCs highly expressed TIMP-1 protein, and the knockdown of TIMP-1 inhibited cell proliferation and arrested cell cycle progression at $\mathrm{G}_{1}$ phase in the ADSCs possibly through the upregulation of $\mathrm{p} 53$, p21, and P-CDK2 protein levels and concurrent downregulation of cyclin E and CDK2 protein levels. These findings suggest that TIMP-1 works as a positive regulator of cell proliferation in ADSCs.
\end{abstract}

\section{Introduction}

Numerous studies have indicated that tissue inhibitors of metalloproteinases (TIMPs) play critical roles in the regulation of extracellular matrix (ECM) metabolism, tissue remodeling, and cellular behavior $[1,2]$. It is widely wellrecognized that TIMPs serve as the inhibitors of matrix metalloproteinases (MMPs) and other metalloproteinases and can regulate their activities and the proteinaceous ECM homeostasis. However, increasing evidence indicates that the range of TIMPs activities is more broader as they can also elicit metalloproteinase-independent biological activities [3, 4]. The human genome has four paralogous genes encoding endogenous inhibitors (TIMP-1, TIMP-2, TIMP-3, and TIMP-4) which all share several structural features. In this respect, TIMP-1 has been shown to be particularly interesting as it not only has a classical role as an inhibitor of MMPs but also has growth factor-like activity $[5,6]$. Additionally, TIMP-1 has been shown to possess other activities in the regulation of various biological processes such as cell growth, differentiation, and apoptosis $[7,8]$.

Previous studies have demonstrated that human bone marrow-derived mesenchymal stem cells (MSCs) constitutively express high level of TIMP-1, and the silencing of TIMP-1 enhances cell migration [9]. Recent studies indicated that TIMP-1 ubiquitously is expressed in numerous human cells and tissues, and it is a cytoprotective released factor from embryonic stem (ES) cells, and its overexpression in ES cells attenuates adverse myocardial remodeling and improves cardiac function in the mice [10]. These findings prompted 
us to hypothesize that TIMP-1 expression may affect the basic functions of stem cells, such as cell proliferation and differentiation. Adipose-derived stem cells (ADSCs) are especially attractive multipotent progenitor cells because they are relatively abundant and more easily acquired. Additionally, ADSCs can differentiate into osteogenic, myogenic, chondrogenic, endothelial, adipogenic, and neural cells in the presence of lineage-specific induction environment [11]. These characteristics endow ADSCs regenerative properties. Therefore, ADSCs are ideal candidates for cell-based therapies in the tissue engineering and regenerative medicine [12]. However, little is known about the expression and function of TIMP-1 in ADSCs. In this study, we investigated whether TIMP-1 can regulate the proliferation of ADSCs. Our findings indicated that TIMP-1 knockdown significantly inhibited cell proliferation and blocked cell cycle progression at $G_{1}$ phase in the ADSCs. The results suggest that TIMP-1 functions as a positive regulator of ADSCs proliferation and may accelerate the application of ADSCs in regenerative medicine.

\section{Materials and Methods}

2.1. Isolation and Culture of ADSCs. ADSCs were isolated from the lipoaspirates of healthy human donors as we described previously $[13,14]$. The written informed consents were obtained from the donors of adipose tissues. This study was reviewed and approved by the Human Research and Ethical Committee of Affiliated Hospital of Guangdong Medical College. The isolated ADSCs were cultured at $37^{\circ} \mathrm{C}$ in low glucose DMEM medium (Gibco, USA) added with $10 \%$ fetal bovine serum (FBS) in a humidified incubator with 5\% $\mathrm{CO}_{2}$, and the cells at passages 4-6 were used for the following experiments.

2.2. Expression Level of TIMP-1 Protein. Expression levels of TIMP-1 protein in adipose tissue, fibroblasts, and ADSCs were detected by western blotting. Briefly, $20 \mu \mathrm{g}$ total protein from each sample was separated on a $10 \%$ SDS polyacrylamide gel and transferred to polyvinylidene difluoride (PVDF) membranes. Subsequently, the membranes were incubated with primary antibodies of TIMP-1 (Abcam, Cambridge, MA) and $\beta$-actin (Abcam) at $4^{\circ} \mathrm{C}$ overnight, followed by incubation with secondary antibody for $2 \mathrm{~h}$ at room temperature. The protein bands were then visualized with an enhanced chemiluminescence system (Amersham Biosciences, NJ, USA).

2.3. Construction of TIMP-1 shRNA Lentiviral Vector and Infection into Cells. The shRNA sequences were used in the following experiments: TIMP-1 shRNA (shTIMP-1): $5^{\prime}$ GTTGTTGCTGTGGCTGATA-3'; the nonsilencing shRNA (mock): $5^{\prime}$-TTCTCCGAACGTGTCACGT-3'. They were, respectively, cloned into the GV248 vector (GeneChem, Guangzhou, China) that contained GFP and puromycin resistance gene. The recombinant virus was packaged in 293T cells using a lentivector expression system (GeneChem) according to the manufacturer's instructions, followed by cellular infection. ADSCs were infected with lentivirusmediated shRNA-TIMP-1 or mock-shRNA when they had grown to about $80 \%$ confluence. The GFP expression level was observed using fluorescence microscopy to determine the infection efficiency. The infected ADSCs were subcultured in puromycin-containing growth medium for 3 days to detect the expression level of TIMP-1 with real-time RT-PCR and western blotting, respectively.

2.4. Quantitative Real-Time RT-PCR. Total RNA was extracted from the ADSCs using TRIzol reagent (Life Technologies, Carlsbad, CA) according to the manufacturer's instructions. The concentration of total RNA was determined by measuring the absorbance at $260 \mathrm{~nm}$. $2 \mu \mathrm{L}$ total RNA was reverse-transcribed into cDNA using oligo $(\mathrm{dT})_{20}$ primer and $\mathrm{MML}-\mathrm{V}$ reverse transcriptase (Takara, Japan), followed by real-time PCR with the reagent SYBR Premix ExTaq ${ }^{\mathrm{TM}}$ (Takara, Japan) on Light Cycler $480^{\circledR}$ real-time PCR system. The PCR primers were used as previously reported [15] and are listed as follows: TIMP-1 forward, 5'-ACTTCCACAGGTCCCACAAC-3'; TIMP-1 reverse, 5'-GCATTCCTCACAGCC AACAG-3'; GAPDH forward, 5'-TGCACCACCAACTGCT TAG-3 ${ }^{\prime}$; GAPDH reverse, $5^{\prime}$-GTTCAGCTCAGGGATGACC- $3^{\prime}$. PCR amplification was conducted at $95^{\circ} \mathrm{C}$ for $3 \mathrm{~min}, 45 \mathrm{cycles}$ at $95^{\circ} \mathrm{C}$ for $30 \mathrm{sec}, 60^{\circ} \mathrm{C}$ for $45 \mathrm{sec}$, followed by $72^{\circ} \mathrm{C}$ for $5 \mathrm{~min}$. The relative expression level of TIMP-1 mRNA was calculated by normalization to the GAPDH mRNA level.

2.5. Cell Proliferation Assay. Cell proliferation was evaluated by MTT assay. Briefly, the cells were plated in 96-well plates at a density of $5 \times 10^{3}$ cells/well and incubated at $37^{\circ} \mathrm{C}$ for 24 , 48,72 , and $96 \mathrm{~h}$ after infection, followed by addition of $10 \mu \mathrm{L}$ MTT solution $(5 \mathrm{mg} / \mathrm{mL})$ to each well for $4 \mathrm{~h}$. The absorbance was recorded at $570 \mathrm{~nm}$ with automated spectrophotometric plate reader (PerkinElmer, USA). The experiments were repeated at least three times.

2.6. Immunocytochemical Staining. Immunocytochemical staining for the proliferative marker, Ki67, was performed as described previously [14], with some modifications. Briefly, the cells were seeded on the glass slides at a density of $5 \times$ $10^{3}$ cells per slide $72 \mathrm{~h}$ after the infection with lentivirusmediated shRNA-TIMP-1 or mock-shRNA. After $24 \mathrm{~h}$ of incubation, the slides were washed twice with PBS and fixed in $4 \%$ paraformaldehyde at room temperature, followed by incubation with a monoclonal Ki67 antibody (1:400; ZSGB-BIO, Beijing, China) for $12 \mathrm{~h}$ at $4^{\circ} \mathrm{C}$. After washing, biotinylated anti-rabbit IgG secondary antibody was added for $20 \mathrm{~min}$ of incubation, followed by incubation with avidin-biotin-peroxidase complex (Vector Laboratories Ltd., Peterborough, UK) for $1 \mathrm{~h}$. Negative control was made without the primary antibody. The slides were evaluated at 400x magnification under the microscope with a digital camera. The percentage of positive Ki67 staining was quantified by ratio of Ki67-positive cells in four different 


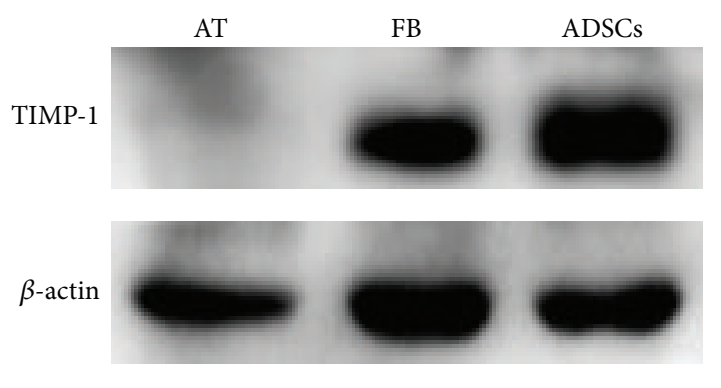

(a)

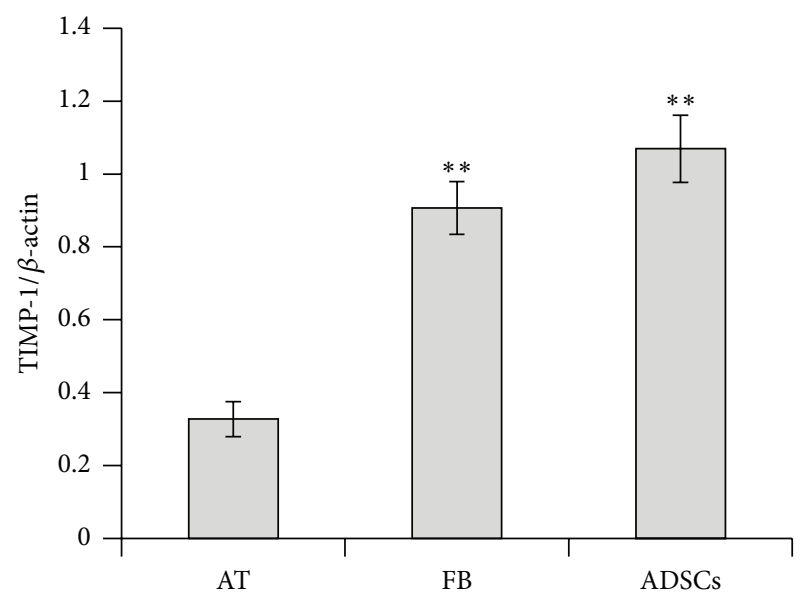

(b)

FIGURE 1: Western blot analysis for TIMP-1 protein expression. The expression levels of TIMP-1 protein in adipose tissue, fibroblasts, and ADSCs were determined by western blotting analysis. (a) The representative western blot images for the bands of TIMP-1. (b) Relative expression levels of TIMP-1 protein were quantified by the densitometry of each band normalized to $\beta$-actin signal. The data represent the mean $\pm \mathrm{SD}(n=3) .{ }^{* *} P<0.01$ versus the AT group. AT: adipose tissue; FB: fibroblasts.

visual areas to total cell number in corresponding areas in each sample.

2.7. Cell Cycle Analysis. Cell were harvested, washed twice with PBS, and fixed in $75 \%$ ethanol for $1 \mathrm{~h}$. The cells were then centrifuged to remove the $75 \%$ ethanol and incubated in cell cycle staining solution containing $50 \mu \mathrm{g} / \mathrm{mL}$ of propidium iodide (Beyotime, China) for $30 \mathrm{~min}$ in the dark at room temperature. The percentage of cells at different phases of cell cycle was determined by flow cytometry (Becton Dickinson, NJ, USA).

2.8. Western Blotting. Protein expression levels of p53, p21, cyclin E, CDK2, and P-CDK2 (CST Company, Danvers, USA) in the parental ADSCs, mock-shRNA ADSCs, and shTIMP1 ADSCs were determined by western blotting according to standard procedures. The intensities of protein bands were quantified by Bio-Rad Quantity One software (Bio-Rad, USA). $\beta$-actin was used as a loading control.

2.9. Statistical Analyses. All values were expressed as mean \pm standard deviation (SD) and analyzed using a SPSS 13.0 statistical package. The comparison between means of two groups was assessed by unpaired Student's $t$-tests. $P<0.05$ was considered statistically different.

\section{Results}

3.1. Expression Level of TIMP-1 Protein in ADSCs. To investigate the possible role of TIMP-1 in ADSCs, we firstly analyzed the levels of TIMP-1 protein expressed in the adipose tissues, fibroblasts, and ADSCs by western blotting. As shown in Figure 1, expression level of TIMP-1 protein was considerably higher in fibroblasts and ADSCs than in the adipose tissue.
3.2. TIMP-1 Knockdown and Its Effects on Cell Proliferation. To elucidate whether TIMP-1 expression has any effect on the proliferation of ADSCs, RNAi was used to generate TIMP1 knockdown cell lines. In this study, lentivirus-mediated infection was performed in ADSCs. The results of RT-PCR confirmed that TIMP-1 mRNA expression in the shTIMP-1 ADSCs was suppressed by about $80 \%$ compared to the cells infected with mock-shRNA (Figure 2(a)). Additionally, western blot analysis demonstrated that TIMP-1 protein expressed in the shTIMP-1 ADSCs was also markedly downregulated (Figures 2(b) and 2(c)), whereas TIMP-1 protein bands were clearly observed in the mock-shRNA cells and parental ADSCs.

On the other hand, MTT assay was performed to evaluate the effect of TIMP-1 knockdown on cell proliferation. Decreased cell proliferation was observed in the shTIMP-1 ADSCs relative to mock-shRNA cells and parental ADSCs (Figure 3), which suggest that TIMP-1 knockdown strongly inhibits the proliferation and growth of ADSCs. To further confirm the inhibitory effect of TIMP-1 knockdown on the proliferation of ADSCs, Ki67 staining was performed. As shown in Figure 4, the percentage of Ki67 positive cells (brownish) was significantly reduced in the shTIMP-1 ADSCs compared with the mock-shRNA control cells or parental ADSCs.

3.3. Effect of TIMP-1 Knockdown on Cell Cycle Progression. To investigate the effect of TIMP-1 knockdown on cell cycle progression, cell cycle distribution of parental ADSCs, mockshRNA ADSCs, and shTIMP-1 ADSCs was analyzed by flow cytometry. As shown in Figure 5, a significantly increased percentage of cells at $\mathrm{G}_{1}$ phase was observed in the shTIMP-1 cells along with a marked decline in the population of $G_{2} / M$ phase cells, which suggests that TIMP-1 knockdown strongly blocked cell cycle progression of ADSCs. 


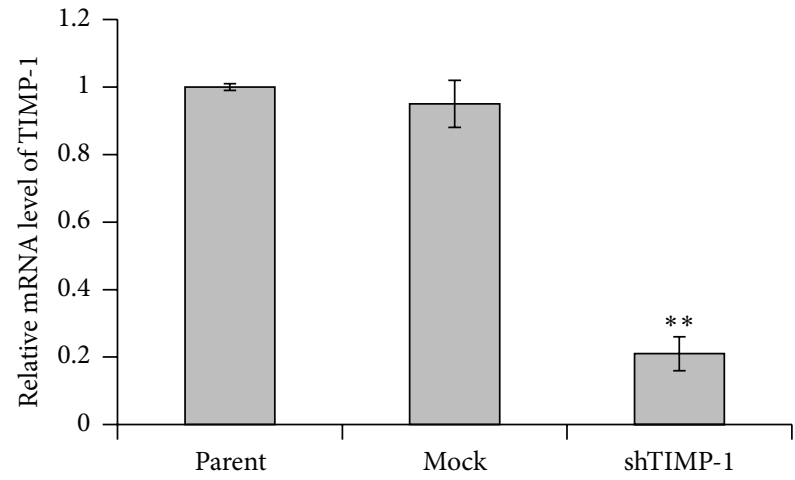

(a)
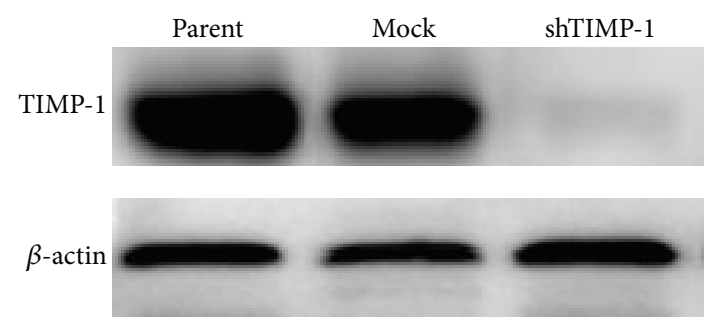

(b)

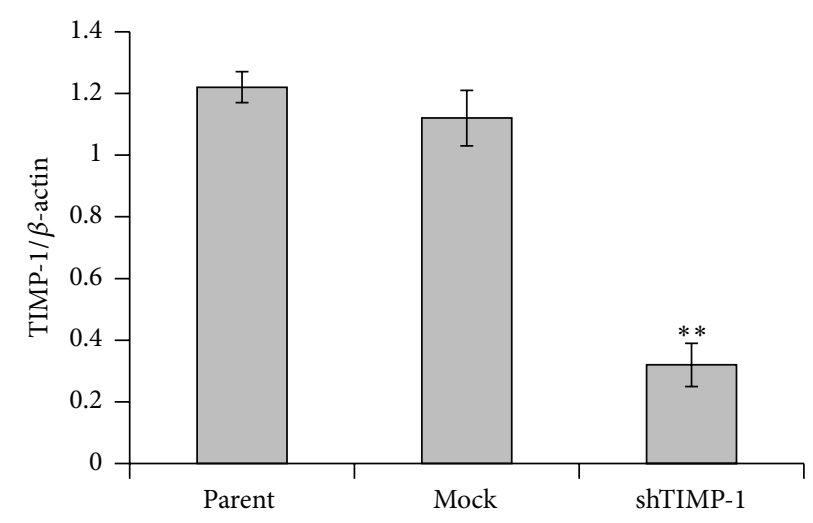

(c)

FIGURE 2: TIMP-1 expression was suppressed by shRNA-TIMP-1. TIMP-1 expression was analyzed by real-time RT-PCR and western blotting, respectively. (a) The levels of TIMP-1 mRNA in parental ADSCs, mock, and shTIMP-1 ADSCs were measured by real-time RT-PCR $72 \mathrm{~h}$ after shRNA infection. TIMP-1 mRNA expression in parental ADSCs is defined as 1 . The $y$-axis represents the normalized TIMP-1 mRNA expression relative to parental ADSCs. (b) Representative western blot images for the detection of TIMP-1 protein expression in the ADSCs infected with shRNA against TIMP-1. (c) The relative expression level of TIMP-1 protein was quantified by the ratio of optical density of TIMP-1 and $\beta$-actin. The data are denoted as mean \pm SD $(n=3) .{ }^{* *} P<0.01$ versus mock-shRNA group. Parental ADSCs: uninfected ADSCs; mock ADSCS: control lentivirus infected ADSCs; shTIMP-1 ADSCs: shRNA-TIMP-1 lentivirus infected ADSCs.

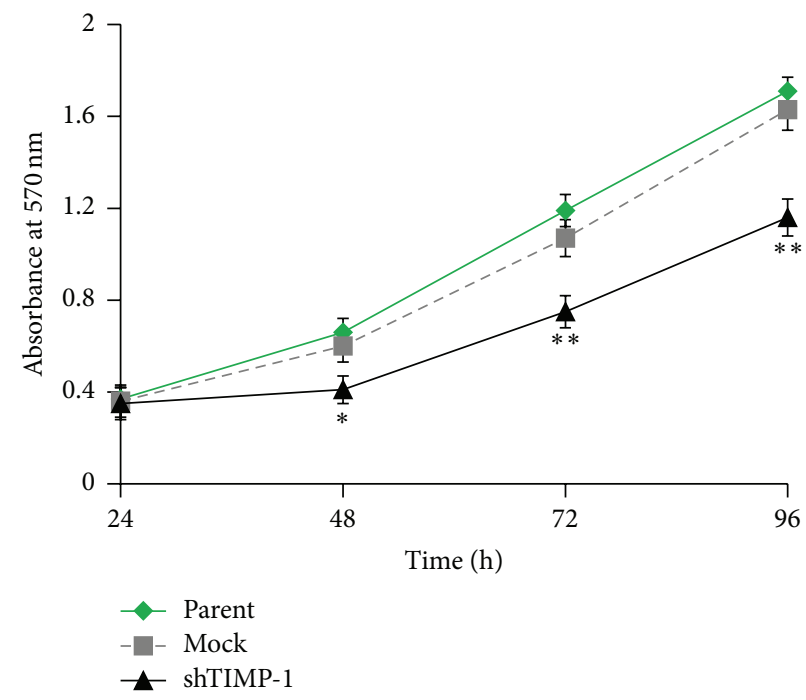

FIGURE 3: siRNA-mediated downregulation of TIMP-1 inhibits the proliferation of ADSCs. Cell growth curves during $96 \mathrm{~h}$ were evaluated by MTT assay. Data are the mean absorbance values of three measurements and the bars represented SD of the mean. ${ }^{*} P<0.05$ and ${ }^{* *} P<$ 0.01 versus mock-shRNA groups. Parent: uninfected ADSCs; mock: control lentivirus infected ADSCs; shTIMP-1: shRNA-TIMP-1 lentivirus infected ADSCs. 


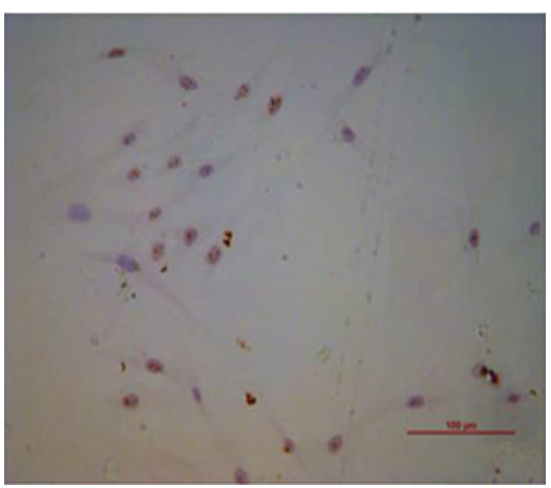

Parent

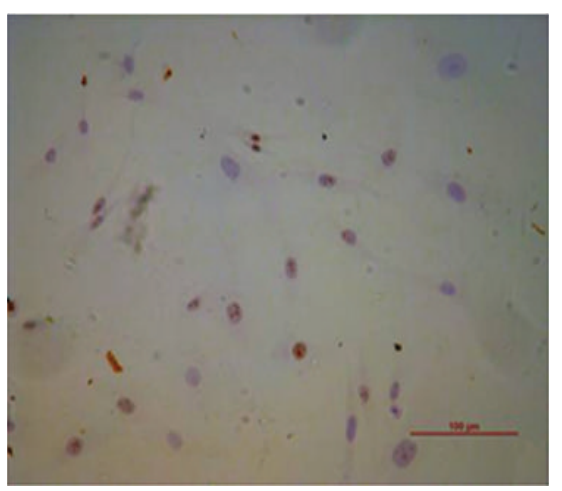

Mock

(a)

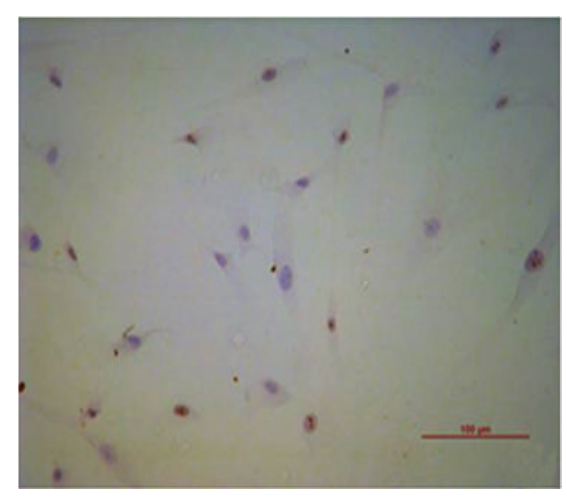

shTIMP-1

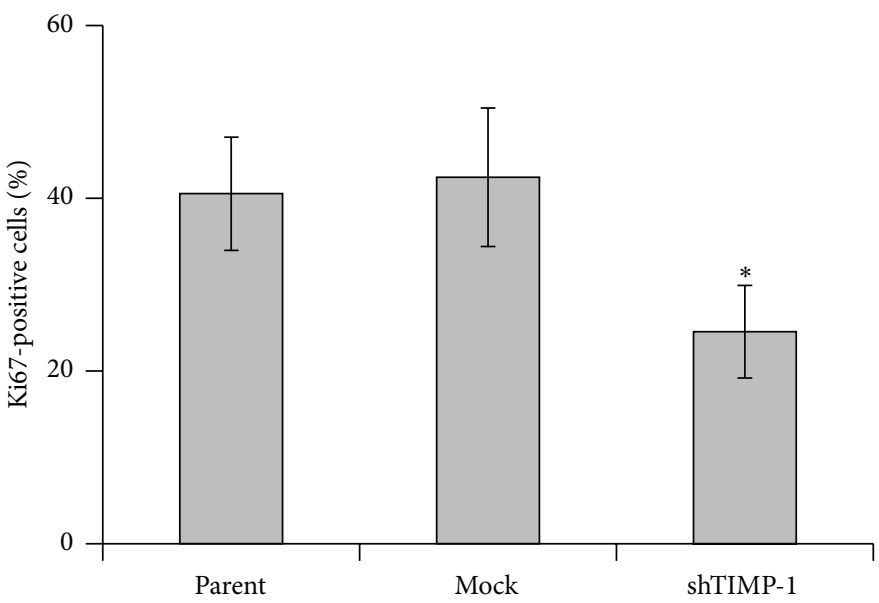

(b)

FIGURE 4: Immunocytochemical staining of Ki67. Ki67 staining was performed to evaluate cell proliferation after TIMP-1 knockdown in the ADSCs. (a) Representative images of Ki67 staining in the parental ADSCs, mock-shRNA ADSCs, and shTIMP-1 ADSCs. (b) Percentage of Ki67 positive cells was quantified in four random areas as described in Section 2. The data are denoted as mean \pm SD $(n=4) .{ }^{*} P<0.05$ versus mock-shRNA groups. Scale bar: $100 \mu \mathrm{m}$.

It is well-known that p53 gene regulates the expression and activity of downstream genes that control cell cycle progression [16]. The levels of p53 and its downstream gene p21 proteins expressed in shTIMP-1 ADSCs were significantly upregulated compared with the control ADSCs (Figure 6). Additionally, the expression level of cyclin E, a relevant cyclin regulating $\mathrm{G}_{1} / \mathrm{S}$ progression, was found to be downregulated after TIMP-1 knockdown in the ADSCs. CDK2 plays a critical role in controlling the progression of cell cycle from $G_{1}$ to $S$ phase [17]. The results of western blotting demonstrated that the level of CDK2 was decreased, but its phosphorylated form was upregulated in the ADSCs after TIMP-1 knockdown.

\section{Discussion}

In this study, we first investigated the expression level of TIMP-1 in ADSCs and found that the constitutive high expression of TIMP-1 was observed in the ADSCs and fibroblasts, and its expression level was very limited in the human adipose tissue. The downregulation of TIMP1 expression induced by specific shRNA in the ADSCs significantly inhibited cell proliferation and arrested cell cycle progression possibly through the regulation of cell cycle-associated protein expression. These findings suggest that TIMP-1 expression promotes cell growth and cell cycle progression of ADSCs.

Since TIMP-1 was discovered about two decades ago as a MMP inhibitor, it has been found to be expressed in various cell types such as fibroblasts, osteoblasts, epithelial and endothelial cells, smooth muscle cells, chondrocytes, mesenchymal stem cells, and many tumor cells $[9,15,18]$. TIMP-1 expression plays various roles in the regulation of cell growth and apoptosis [3]. It was reported that TIMP-1 expression promoted epithelial cell proliferation during mouse mammary development [19]. But other studies demonstrated that TIMP-1 reduced the growth rate of human breast epithelial cells (MCF10A) by inducing cell cycle arrest at $G_{1}$ phase [20]. These conflicting data suggest that TIMP-1 expression results in different effects on cell growth in various cell types. 

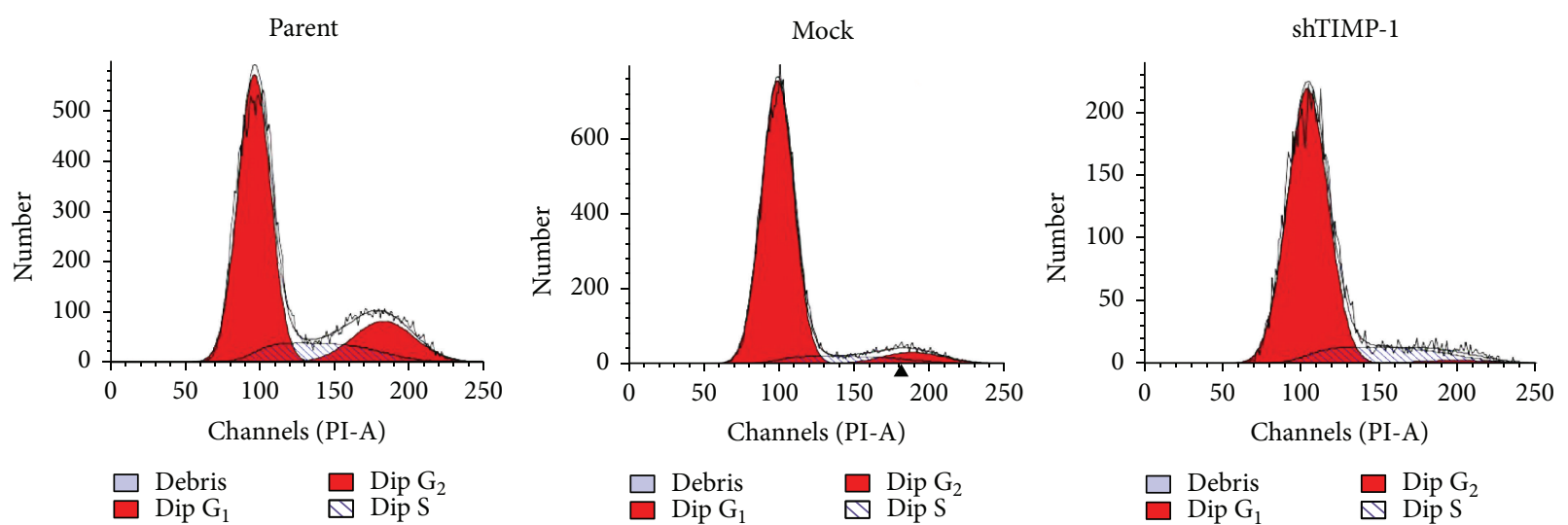

(a)

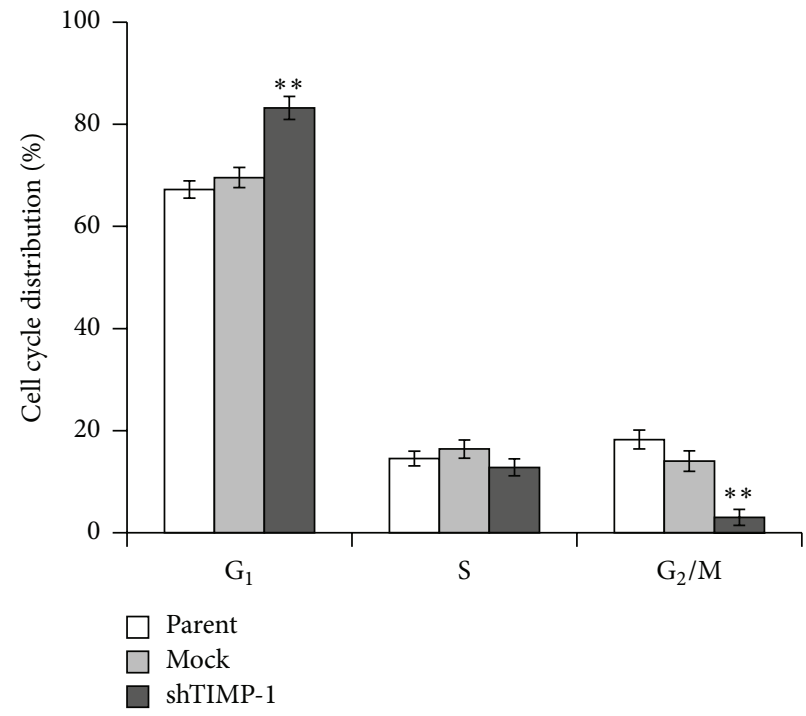

(b)

Figure 5: TIMP-1 knockdown blocked cell cycle progression. Cells were stained with propidium iodide and cell cycle distribution was then analyzed using flow cytometry $72 \mathrm{~h}$ after infection. (a) Representative images of cell cycle distribution in parental, mock-shRNA, and shTIMP1 ADSCs. (b) The histogram was the statistical data from three independent experimental replicates. ${ }^{* *} P<0.01$ versus the mock-shRNA groups.

The specific effects of the TIMPs on cell fate probably depend on the cell context and specific model system under study [4].

In the present study, TIMP-1 expression was successfully downregulated in the ADSCs through lentivirus-mediated shRNA against TIMP-1. MTT assay and Ki67 staining both showed that cell proliferation was significantly suppressed in the shTIMP-1 ADSCs, which was possibly associated with cell cycle arrest. The results of flow cytometry analysis confirmed cell cycle arrest at $G_{1}$ phase in the ADSCs after TIMP-1 knockdown. It is well-known that p53 plays an important role in the regulation of cell cycle arrest, senescence, cell metabolism, apoptosis, or autophagy [21]. In this study, the expression levels of p53 and p21 proteins were both significantly upregulated when TIMP-1 was silenced in the ADSCs. p21 is one of the downstream genes of p53, and it has been found to modulate cell cycle progression at $G_{1} / S$ phase transition [22]. Thus, our results indicated that TIMP1 knockdown resulted in cell cycle arrest at $G_{1}$ phase and reduced the percentage of $S$ and $G_{2} / M$ phase cells possibly through the modulation of p53-p21 signaling pathway. This is similar to previous reports that TIMP-1 reduced the growth rate of the hepatocellular carcinoma cells (BEL-7402) by upregulation of p21 [23]. Additionally, p21 is a negative regulator of cyclin-dependent kinase (CDK) inhibitor and can interact with $\mathrm{CDK}$ including $\mathrm{CDK} 2$ complexes that control the $\mathrm{G}_{1} / \mathrm{S}$ phase transition resulting in cell cycle arrest $[24,25]$. Therefore, we detected the expression levels of cyclin $\mathrm{E}$ and $\mathrm{CDK} 2$ proteins in the ADSCs after TIMP-1 knockdown. The results indicated that shRNA-mediated downregulation of TIMP-1 significantly reduced the expression levels of cyclin $\mathrm{E}$ and $\mathrm{CDK} 2$ proteins but increased the production of CDK2 phosphorylated forms in the shTIMP-1 ADSCs, which at least partly contributed to cell cycle arrest in these cells.

In conclusion, we have demonstrated that TIMP-1 downregulation by shRNA against TIMP-1 gene significantly inhibited cell proliferation and induced cell cycle arrest at $G_{1}$ phase 


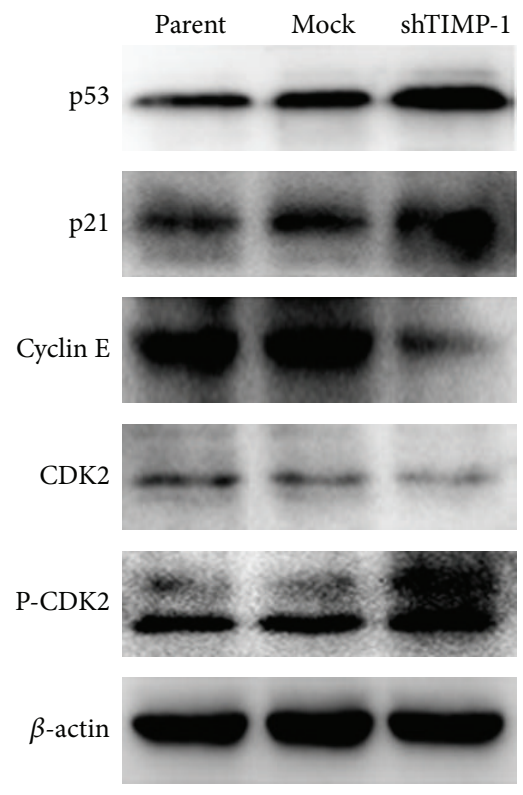

(a)

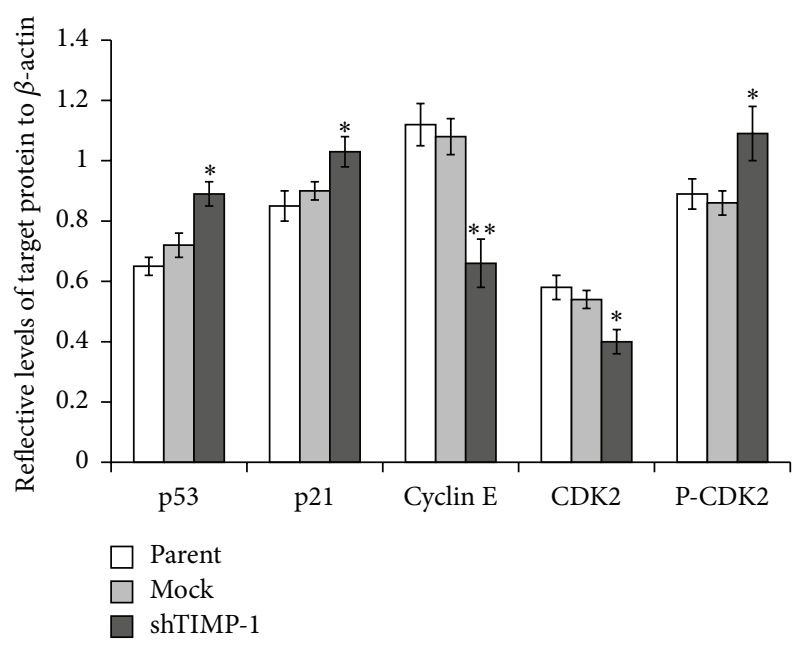

(b)

FIGURE 6: Effects of TIMP-1 knockdown on p53 protein and cell cycle biomarkers. The levels of p53, p21, cyclin E, CDK2, and P-CDK2 proteins were analyzed by western blotting after TIMP-1 knockdown. (a) The representative western blot images. (b) The expression levels of p53, p21, cyclin $\mathrm{E}, \mathrm{CDK} 2$, and P-CDK2 were quantified by the densitometry of each band and were normalized by internal loading control, $\beta$-actin. The data represent the mean $\pm \mathrm{SD}(n=3) .{ }^{*} P<0.05$ and ${ }^{* *} P<0.01$ versus the mock-shRNA groups. Parent: uninfected ADSCs; mock: control lentivirus infected ADSCs; shTIMP-1: shRNA-TIMP-1 lentivirus infected ADSCs.

in the ADSCs possibly through upregulating the expression levels of $\mathrm{p} 53$, p21, and $\mathrm{P}-\mathrm{CDK} 2$ and reducing the production of cyclin $\mathrm{E}$ and $\mathrm{CDK} 2$ proteins. These findings suggest that TIMP-1 is a positive regulator of cell growth in human ADSCs.

\section{Competing Interests}

All the authors declare that they have no competing interests.

\section{Authors' Contributions}

Peihua Zhang and Jin Li contributed equally to this work.

\section{Acknowledgments}

This work was partly supported by Natural Science Foundation of Guangdong Province (Grant no. 2014A030313535), the National Natural Science Foundation of China (81372511 to Xudong Tang), Zhanjiang Municipal Governmental Specific Financial Fund Allocated for Competitive Scientific \& Technological Projects (no. 2014A06005 and no. 2014C01022), and the Scientific Research Starting Foundation for the Doctors of Guangdong Medical University (Grant nos. B2012036 and BJ201510).

\section{References}

[1] C. Montagnani, F. Le Roux, F. Berthe, and J.-M. Escoubas, " $\mathrm{Cg}$-TIMP, an inducible tissue inhibitor of metalloproteinase from the Pacific oyster Crassostrea gigas with a potential role in wound healing and defense mechanisms," FEBS Letters, vol. 500, no. 1-2, pp. 64-70, 2001.

[2] B. Nagy, L. Szélig, S. Rendeki et al., "Dynamic changes of matrix metalloproteinase 9 and tissue inhibitor of metalloproteinase 1 after burn injury," Journal of Critical Care, vol. 30, no. 1, pp. 162166, 2015.

[3] K. Brew and H. Nagase, "The tissue inhibitors of metalloproteinases (TIMPs): an ancient family with structural and functional diversity," Biochimica et Biophysica Acta, vol. 1803, no. 1, pp. 555-571, 2010.

[4] W. G. Stetler-Stevenson, "Tissue inhibitors of metalloproteinases in cell signaling: metalloproteinase-independent biological activities," Science Signaling, vol. 1, no. 27, p. re6, 2008.

[5] T. Hayakawa, K. Yamashita, K. Tanzawa, E. Uchijima, and K. Iwata, "Growth-promoting activity of tissue inhibitor of metalloproteinases-1 (TIMP-1) for a wide range of cells. A possible new growth factor in serum," FEBS Letters, vol. 298, pp. 29-32, 1992.

[6] A. J. Fowell, J. E. Collins, D. R. Duncombe, J. A. Pickering, W. M. C. Rosenberg, and R. C. Benyon, "Silencing tissue inhibitors of metalloproteinases (TIMPs) with short interfering RNA reveals a role for TIMP-1 in hepatic stellate cell proliferation," Biochemical and Biophysical Research Communications, vol. 407, no. 2, pp. 277-282, 2011

[7] N. Boudreau, C. J. Sympson, Z. Werb, and M. J. Bissell, "Suppression of ICE and apoptosis in mammary epithelial cells by extracellular matrix," Science, vol. 267, no. 5199, pp. 891-893, 1995.

[8] L. Guedez, L. Courtemanch, and M. Stetler-Stevenson, "Tissue inhibitor of metalloproteinase (TIMP)-1 induces differentiation 
and an antiapoptotic phenotype in germinal center B cells," Blood, vol. 92, no. 4, pp. 1342-1349, 1998.

[9] C. Ries, V. Egea, M. Karow, H. Kolb, M. Jochum, and P. Neth, "MMP-2, MT1-MMP, and TIMP-2 are essential for the invasive capacity of human mesenchymal stem cells: differential regulation by inflammatory cytokines," Blood, vol. 109, no. 9, pp. 4055-4063, 2007.

[10] C. Glass and D. K. Singla, "Overexpression of TIMP-1 in embryonic stem cells attenuates adverse cardiac remodeling following myocardial infarction," Cell Transplantation, vol. 21, no. 9, pp. 1931-1944, 2012.

[11] H. C. Huang, Y. J. Chang, W. C. Chen, H. I. Harn, M. J. Tang, and C. C. Wu, "Enhancement of renal epithelial cell functions through microfluidic-based coculture with adiposederived stemcells," Tissue Engineering Part A, vol. 19, no. 17-18, pp. 2024-2034, 2013.

[12] A. Faroni, R. J. P. Smith, and A. J. Reid, "Adipose derived stem cells and nerve regeneration," Neural Regeneration Research, vol. 9, no. 14, pp. 1341-1346, 2014.

[13] G. Zeng, K. Lai, J. Li et al., "A rapid and efficient method for primary culture of human adipose-derived stem cells," Organogenesis, vol. 9, no. 4, pp. 287-295, 2014.

[14] J. Li, G. Zeng, Y. Qi et al., "Xenotransplantation of human adipose-derived stem cells in zebrafish embryos," PLoS ONE, vol. 10, no. 4, Article ID e0123264, 2015.

[15] M. Aoki, K. Miyake, R. Ogawa et al., "siRNA knockdown of tissue inhibitor of metalloproteinase-1 in keloid fibroblasts leads to degradation of collagen type I," Journal of Investigative Dermatology, vol. 134, no. 3, pp. 818-826, 2014.

[16] G. Zeng, J. Liu, H. Chen et al., "Dihydromyricetin induces cell cycle arrest and apoptosis in melanoma SK-MEL-28 cells," Oncology Reports, vol. 31, no. 6, pp. 2713-2719, 2014.

[17] J. Li, M. Deng, Q. Wei, T. Liu, X. Tong, and X. Ye, "Phosphorylation of MCM3 protein by cyclin E/cyclin-dependent kinase 2 (Cdk2) regulates its function in cell cycle," The Journal of Biological Chemistry, vol. 286, no. 46, pp. 39776-39785, 2011.

[18] E. Lambert, E. Dassé, B. Haye, and E. Petitfrère, "TIMPs as multifacial proteins," Critical Reviews in Oncology/Hematology, vol. 49, no. 3, pp. 187-198, 2004.

[19] J. E. Fata, K. J. Leco, R. A. Moorehead, D. C. Martin, and R. Khokha, "Timp-1 is important for epithelial proliferation and branching morphogenesis during mouse mammary development," Developmental Biology, vol. 211, no. 2, pp. 238-254, 1999.

[20] M. E. Taube, X.-W. Liu, R. Fridman, and H.-R. C. Kim, “TIMP1 regulation of cell cycle in human breast epithelial cells via stabilization of $27^{\mathrm{KIPl}}$ protein," Oncogene, vol. 25 , no. 21 , pp. 3041-3048, 2006.

[21] J. P. Kruse and W. Gu, "Modes of p53 regulation," Cell, vol. 137, no. 4, pp. 609-622, 2009.

[22] M. J. Armstrong, M. T. Stang, Y. Liu et al., "Interferon Regulatory Factor 1 (IRF-1) induces p21 WAF1/CIP1 dependent cell cycle arrest and p21 WAF1/CIP1 independent modulation of survivin in cancer cells," Cancer Letters, vol. 319, no. 1, pp. 56-65, 2012.

[23] S. Y. Guo, X. Shen, J. Yang et al., "TIMP-1 mediates the inhibitory effect of interleukin- 6 on the proliferation of a hepatocarcinoma cell line in a STAT3-dependent manner," Brazilian Journal of Medical and Biological Research, vol. 40, no. 5, pp. 621-631, 2007.

[24] A. B. Niculescu III, X. Chen, M. Smeets, L. Hengst, C. Prives, and S. I. Reed, "Effects of p $21^{\mathrm{Cip1} / \text { Wafl }}$ at both the $\mathrm{G}_{1} / \mathrm{S}$ and the $\mathrm{G}_{2} / \mathrm{M}$ cell cycle transitions: $\mathrm{pRb}$ is a critical determinant in blocking DNA replication and in preventing endoreduplication," Molecular and Cellular Biology, vol. 18, no. 1, pp. 629-643, 1998.

[25] V. V. Ogryzko, P. Wong, and B. H. Howard, "WAF1 retards Sphase progression primarily by inhibition of cyclin-dependent kinases," Molecular and Cellular Biology, vol. 17, no. 8, pp. 48774882, 1997. 

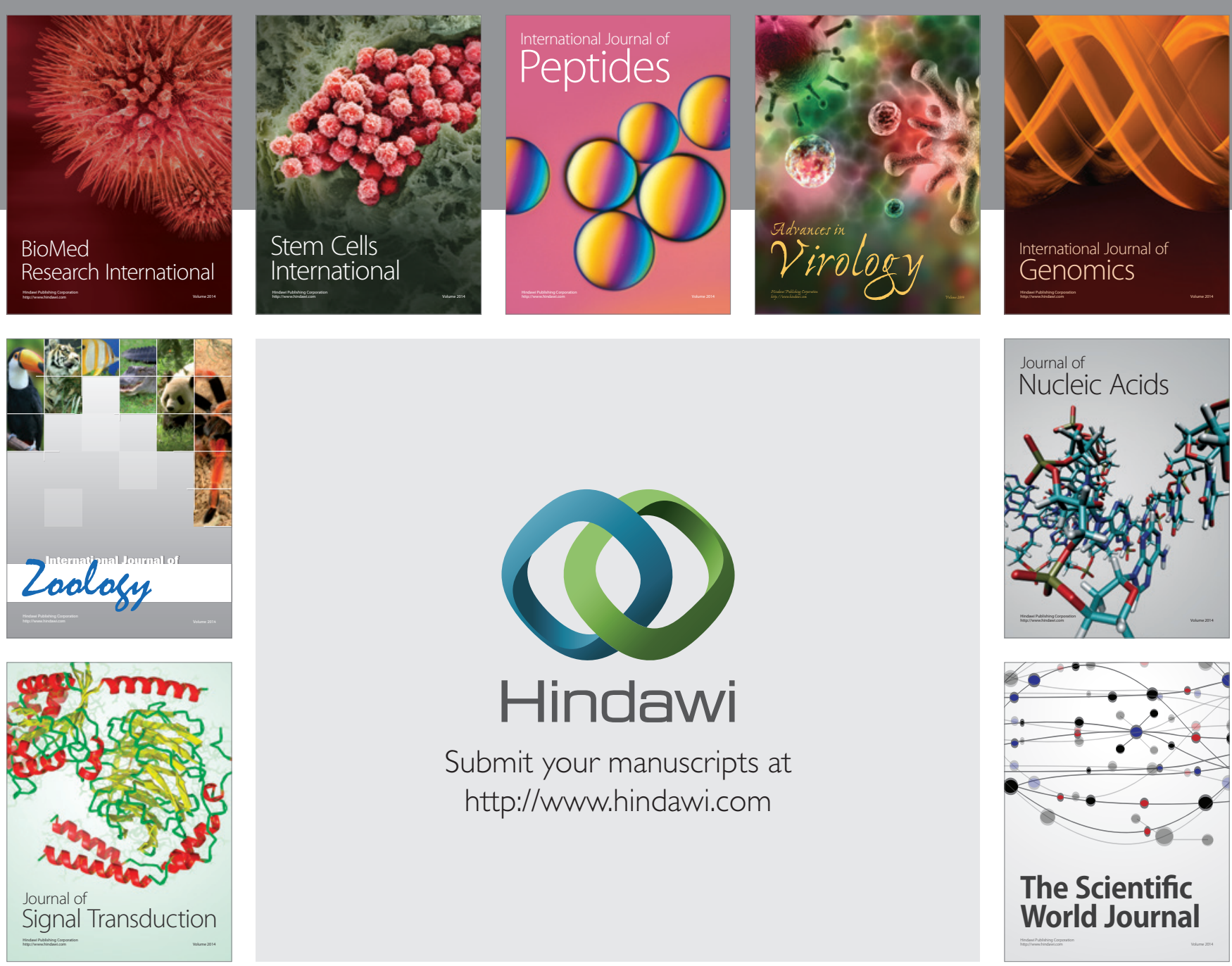

Submit your manuscripts at

http://www.hindawi.com
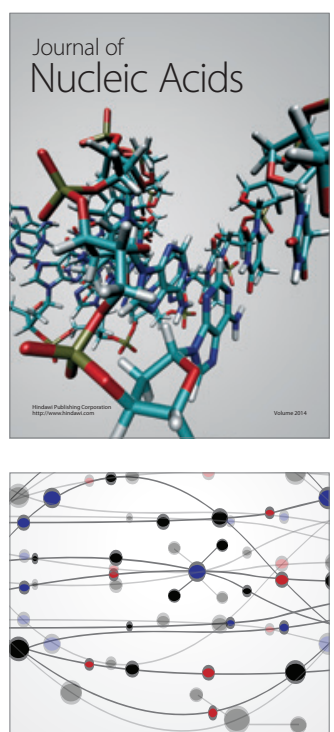

The Scientific World Journal
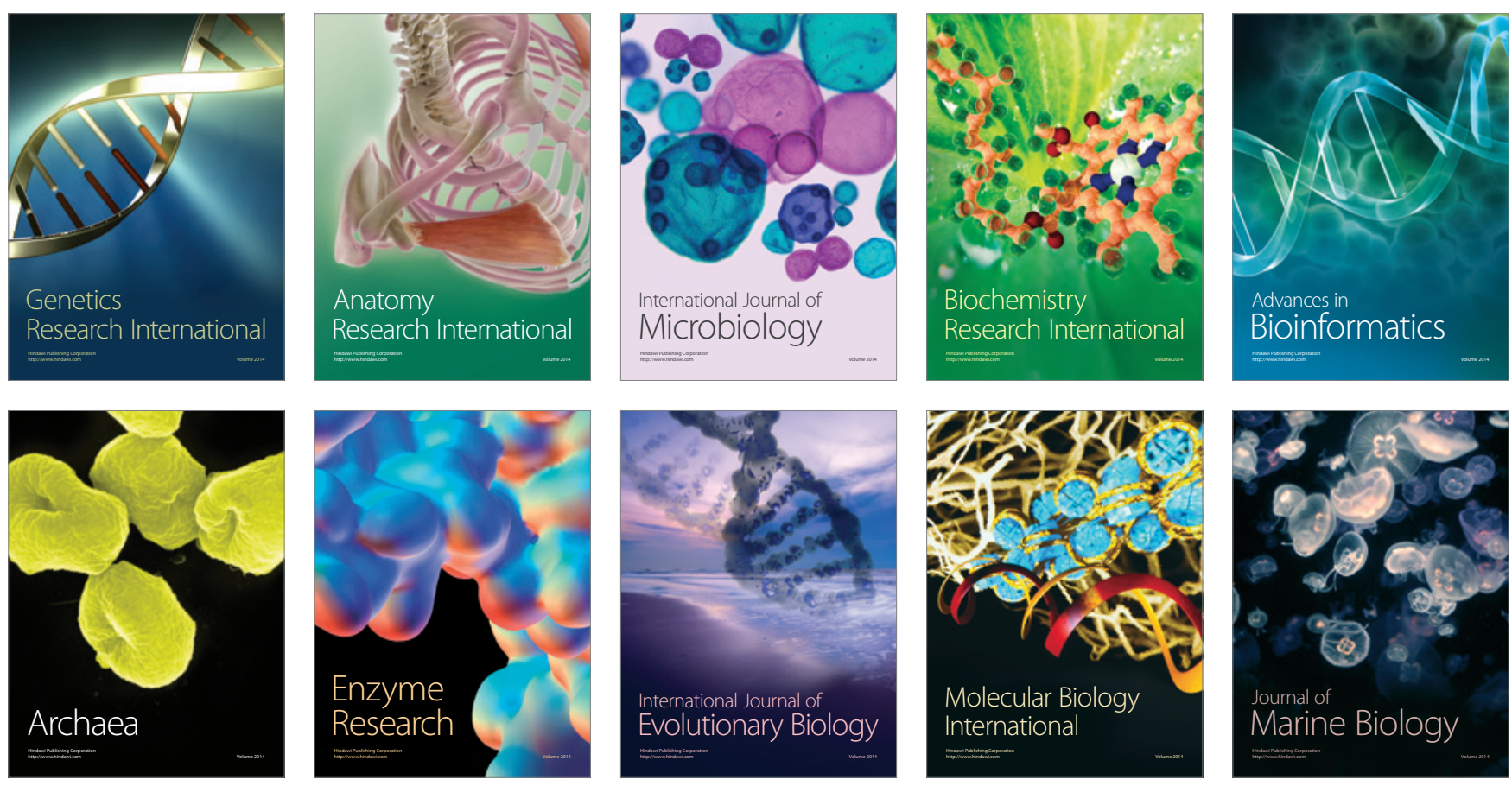